\title{
A Rendering-Based CAD Method For The Design And Testing Of Rearview Mirrors
}

\author{
Monica Carfagni and Luca Landi \\ Dipartimento di Meccanica e Tecnologie Industriali, Università di Firenze, Italy \\ email: landil@DMTI.unifi.it
}

\begin{abstract}
A computer-aided design (CAD) method for modelling rearview mirrors and simulating their visibility is presented. The application was implemented on automatic modelling and rendering modules of a commercial CAD-CAE program.

Through the use of ray tracing and modelling techniques, the designsimulation phase was dramatically reduced to less than ten minutes, with maximum visibility achievable on the first try. In addition to the tests prescribed by current international standards, special procedures were developed to optimise and test visibility. The method, which is applicable to a broad range of vehicles, is currently being used by an automobile manufacturer.
\end{abstract}

Key words: Geometric Modelling, Industrial Application, Virtual Prototyping

\section{INTRODUCTION}

Computer-aided design (CAD) methods, which are dramatically reducing new products' time to market, especially in the final development and test phases, are increasingly being applied to solve specific design problems (Wheatley 1999, Su 1999). In this work, a CAD method was developed to design rearview mirrors and simulate their visibility.

The manufacturer's CAD software, Catia, developed by Dassault Systèmes, has allowed custom routines to be seamlessly integrated with the main software. The routines are able to use the numerous already existing $\mathrm{CAD} / \mathrm{CAM} / \mathrm{CAE}$ function libraries. In addition, Catia's built-in ray-tracing 
modulus, originally developed for multimedia presentations and for texture simulations and used in lieu of a conventional simulation method, is proving to be an effective tool for simulating visibility (Carfagni et al. 1997, IBM 1997).

The optical law of flat and spherical surface reflections is the underlying principle of the method. When a beam of light travelling in a medium encounters the separation surface with another medium, a part of the incident beam is reflected back. The reflection of parallel luminous rays on a smooth surface (i.e., one whose roughness is less than the wavelength of the incident light) produces the so-called "mirror-image reflection," since, after being reflected, it retains its parallel structure, whereas the reflection of parallel light rays on a rough surface causes them to exit the separation surface in different directions so that no mirror image results. In this work, "reflection" is used to mean "mirror-image reflection." Hence, a single ray of light incident a surface by angle $\theta_{1}$ will reflect from the mirror by reflection angle $\theta_{2}$ as illustrated in Figure 1.

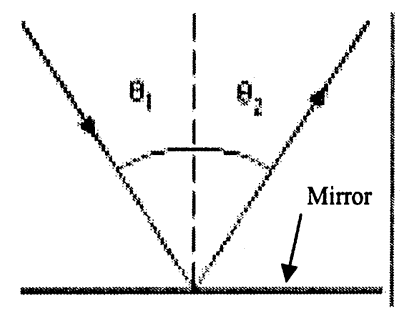

Figure 1. Ray of incident and reflecting light on a mirror

On the basis of the law of optics, we can write $\theta_{1}=\theta_{2}$. Let us consider a point source of light, $O$, placed at distance $s_{1}$ from a flat mirror (Figure 2).

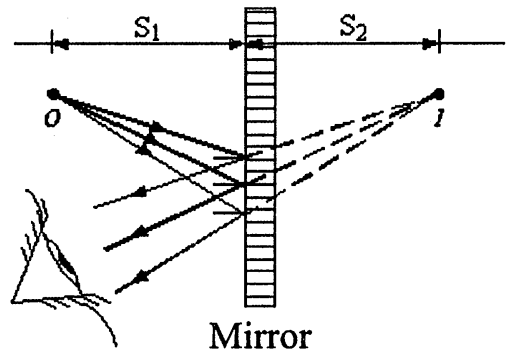

Figure 2. Definition of the image of an object reflected in a flat mirror

After being reflected, the rays diverge and, to an observer, appear to come from image point, $l$, lying distance $s_{2}\left(s_{2}=s_{1}\right)$ from the mirror. The resulting image located in $O$ is called the "object image". The object image of a dimensionless object formed by a flat mirror lying on a plane normal to 
the mirror is symmetrical to the object with respect to the mirror (Born and Wolf 1980).

Moreover, if the light actually passes through the image point (as happens in concave mirrors), we term the resulting image "real"; conversely, if the light does not actually intersect the image point, we term the image "virtual" (as in the case of convex and flat mirrors). From the observer's viewpoint, we can consider an object reflected in a mirror either through a "real eye" or a "virtual eye" (the latter belonging to a virtual observer behind the mirror, which thus represents a transparent viewing pane) (Figure 3). The sole difference in the real and virtual images is that the virtual image is horizontally reversed which, however, has no effect on our investigation.

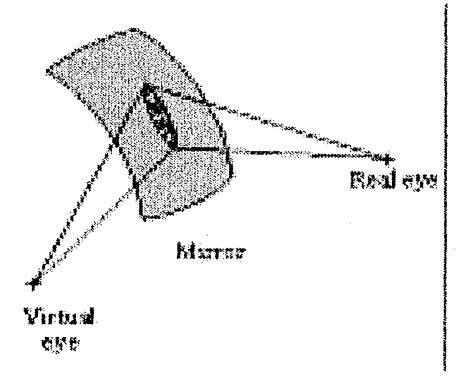

Figure 3. The real eye and the virtual eye

The above considerations apply to flat mirrors such as internal rearview mirrors. Conversely, external mirrors are generally convex and spherical.

The convex spherical mirror is defined by curvature radius, $R$, from the centre, $C$, of the sphere containing the mirror and by the midpoint of the mirror outline on plane, $V$. The straight line passing through $C$ and $V$ is the mirror's main axis (Figure 4).

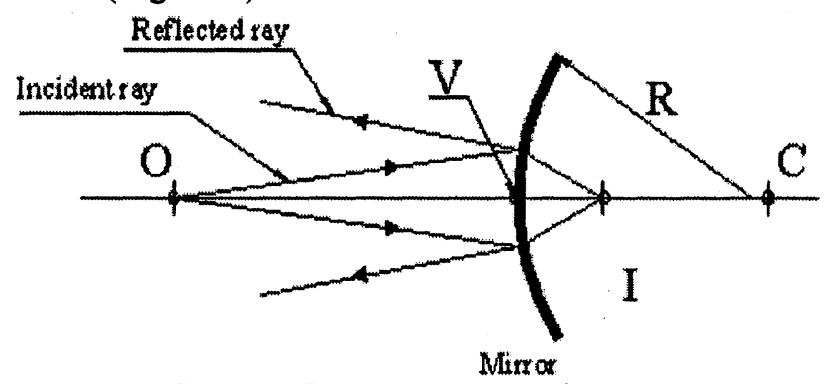

Figure 4. Images on convex mirrors

Let us now consider a point light source on point $O$ of the main axis where distance $O V$ exceeds distance $V C$, as in the case of objects far from the test vehicle. The reflected rays converge and meet in image point $I$, and continue to diverge as if a virtual point source existed in $I$. The result, in keeping with the definition in the preceding paragraph, is a virtual image. 
The rays that diverge from the source $O$ are called "paraxial rays", since the angle they form with the main axis is small compared to the full mirror aperture angle (for clarity, the constructions in Figure 4 contain deliberately oversize parallaxis angles).

If the incident rays diverge excessively from the main axis, they fail to produce a single image point, which means the image appears out of focus. The phenomenon of the distortion of a reflected image according to mathematical laws, termed "spherical aberration," occurs to some extent in all spherical mirrors (Lunemberg, 1944, Born and Wolf 1980). In this work, spherical aberration becomes increasingly important as objects get closer and closer to the test vehicle.

From the above considerations, it can be seen that, since the rays reflected by a convex mirror are always divergent, the use of a convex mirror provides a greater rear visibility range. Hence, passenger vehicles are usually equipped with one internal flat mirror and two external convex mirrors with $R \approx 2000 \mathrm{~mm}$.

\section{STANDARDS FOR REARVIEW VISIBILITY}

The rearview mirrors designed according to the proposed procedure were tested according to the manufacturer's standards that apply European Union standards EEC 71/127, EEC 79/795, and EEC 82/205. The standard prescribing the procedures for visibility testing on full-scale prototypes ensures a minimum visibility range for the driver dependent upon the tested mirror (Fiat 1997 and 1996). Hence, the overlap of the driver's visibility range should contain a minimum visibility range defined by the standard. To accomplish this, we implemented the CAD with three different surfaces having three different visibility ranges.

The rectangular surface used for the internal mirror tests, termed "visibility barrier," is illustrated in Figure 5.

The size of the visibility barrier is related to the distance between the driver's eyes and surfaces. The barriers in the standards were developed according to precise perspective relationships that maintain the driver's visibility range unaltered despite variances in the distance between the driver's eye and the barrier. The standard also indicates that the presence of objects such as headrests, window shade supports, rear windshield wipers, and antennas can further reduce the visibility range up to $15 \%$ of the prescribed visual range in the case of internal mirrors and up to $10 \%$ in the case of external mirrors. 


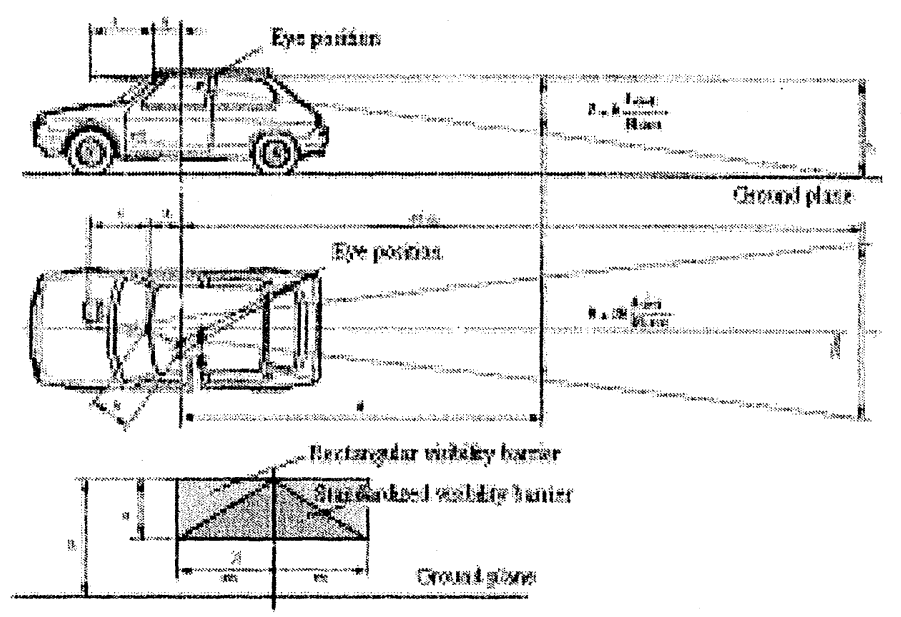

Figure 5. Test environment for an internal mirror

The test surface defined by the standard is triangular, whereas we have defined a rectangle whose base and height respectively equal the base and height of the triangle in the standard. This definition of visibility was determined jointly with the manufacturer with the aim of maximising safety.

\section{PROCEDURE}

Having successfully concluded the feasibility study with Catia (Carfagni et al. 1997), we then developed the simulation technique and the interactive procedures for designing the mirrors and simulating their visibility (Kolb, 1995). The design procedure comprises six steps:

Step 1. Modelling the mirrors. In this step, the CAD automatically models the mirrors with the data input by the designer via a user-friendly interface built into the CAD system.

Step 2. Correcting the models. In this step, the corrections to be made to the CAD models are automatically calculated according to the vehicle configuration and the orientation of the mirror.

Step 3. Modelling the visibility barriers. In this step, the barriers for the visibility tests are automatically modelled.

Step 4. Simulating visibility. In this step, the simulation parameters (chamber, light sources, test environment, and component textures) are derived and input.

Step 5. Performing special testing. In this step, additional testing of components such as heated rear windows that are not indicated in the standards are carried out. 
Step 6. Saving and storing data. In this step, the test images and design data are saved and stored according to the manufacturer's in-house standards.

In addition, a detailed User's Manual is provided to guide the designer step-by-step through the procedures. It is especially useful in Step 4, where the designer is forced to use explicit CAD commands owing to the limitations in the automatic function libraries available to the rendering module.

The first three steps were implemented through the creation of special routines, which were developed with Catia's Interactive User Access and integrated in the CAD procedure (IBM 1997). A detailed description of the procedure follows.

Step 1. Modelling the mirrors. Data for the modelling and visibility test phases are input via two specially designed panels, one for the input of geometric data and one for the selection of special tests not indicated in the standards. (These tests will be discussed later on.) The required inputs are:

- dimensions, outline patterns, and the position of the mirror and joint

- the driver's position point corresponding to the joint between the mannequin's back and hip

- data regarding the test vehicle configuration

- geometric data regarding the visibility barrier

As was previously mentioned, the flexibility of the routines enables modelling of any type of symmetrical rearview mirror (internal or external, flat or spherical, with straight or curved edges or combinations thereof). Only slight changes to the standard procedures are required to test asymmetrical mirrors (Carfagni and Landi 2000). As the data are being input, the procedure checks for dimensional consistency to prevent the system from crashing during the modelling and/or orientation steps. The dimensional value range is extremely broad, allowing the design and testing of mirrors with semi-widths and -heights up to $50 \mathrm{~cm}$.

Step 2. Correcting the models. In addition to geometry, rear visibility depends upon random factors that are difficult to predict such as variations in vehicle configuration in relation to load and the amount of air in the tires. The proposed method accounts for these factors by determining vehicle configuration on the basis of the following data:

- the midpoint dimensions of the front wheel

- the radius of the front wheels when loaded

- the vehicle pitch

- the height from the ground of the rear wheel midpoint

- the radius of the rear wheels when loaded.

If the configuration inputs are unknown, the test can still be carried out by insertion of a vehicle pitch of less than $100 \mathrm{~mm}$, which annuls the 
calculation of the configuration-derived corrections. The method automatically calculates the position corrections in relation to the input parameters and automatically determines the optimal orientation of the rear mirror to be tested. Having input a system of spherical coordinates by means of two longitudinal and latitudinal angles with respect to the mirror's fixed joint, we can search for the optimum visibility angles (Figure 6).

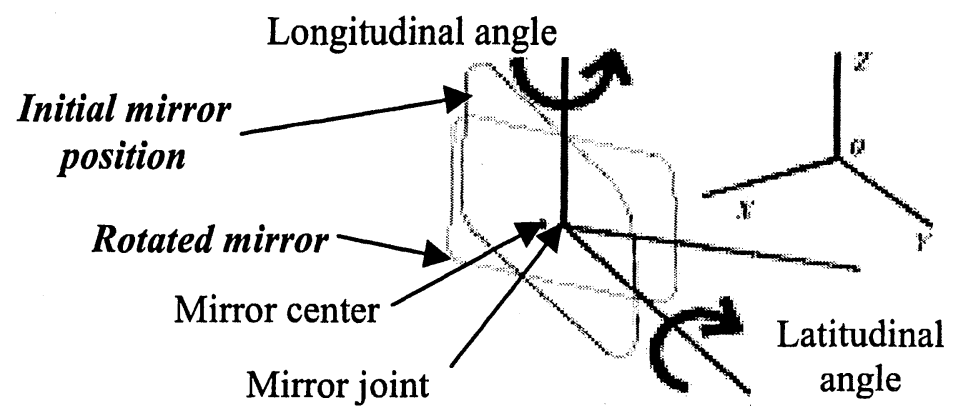

Figure 6. Rear-view mirror with positive longitudinal and latitudinal directions

Automatic orientation has two main objectives:

1. To provide focus on the inside of the mirror for as much of the visibility barrier as possible, while, at the same time, ensuring the best possible trade-off between the driver's right and left vision

2. To mathematically account for the distortion of the reflected image owing to the possible presence of spherical mirrors and thus of spherical aberration without having to conduct preliminary visibility tests.

As human vision does not allow the position of the rearview mirror to be optimised for both of the driver's eyes, a new entity, or "central eye", was defined for the iterative process of optimising the longitudinal and latitudinal angles. The central eye represents the point equidistant between the driver's real eyes. With the central eye, it is possible to achieve the "mean optimum orientation," i.e., a compromise between the vision furnished by the driver's real eyes (Sivak et al. 1997, Nagata 1998). The results obtained with this approximation have been very satisfactory as we proved with some tests. The orientation procedure comprises two iterative cycles. Both are stable and convergent in a broad range of actual orientation situations, and in all theoretical cases perfectly comply with all design and simulation requirements.

Iterative cycle \#1. The first iterative cycle orients the rearview mirror, using the two vectors shown in figure 7, so that the central eye "views" the midpoint of the visibility barrier in the middle of the mirror (we must obtain $a_{1}=a_{2}$ at the end). Starting from zero longitudinal and latitudinal angles, the routine continues until the difference between the two angles obtained from two successive iteration drops to below $0.005 \mathrm{deg}$. The stereoscopic views 
approach is not feasible using Catia because the insertion of the gap between the pilot's eyes causes a pilot's head rotation prohibited by international rearview testing standards.

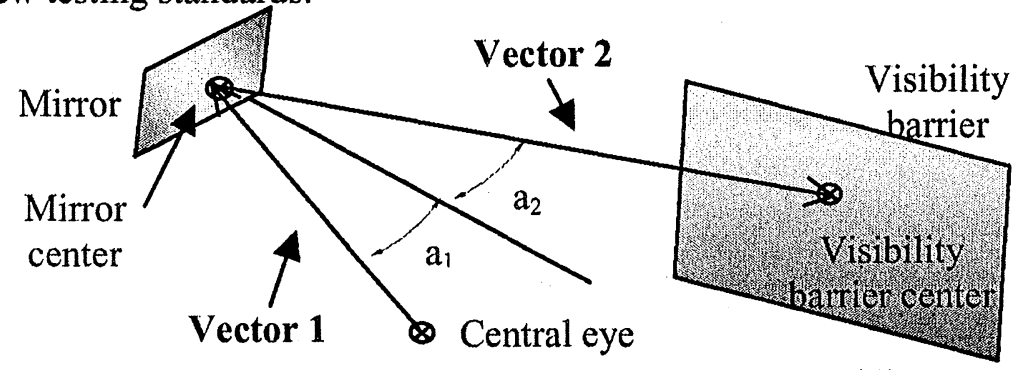

Ground line

Figure 7. Iterative cycle \#1 for mirror optimal orientation

Iterative cycle \#2. Using the longitudinal and latitudinal values furnished by the first cycle, the second cycle superimposes the greatest possible area of the visibility barrier on the test mirror. The second cycle provides the mirror's final orientation by projecting the visibility barrier on the test mirror, using the virtual eyes as the vanishing point. To account for aberration, spherical mirrors must be defined as equivalent flat mirrors (Figure 8).

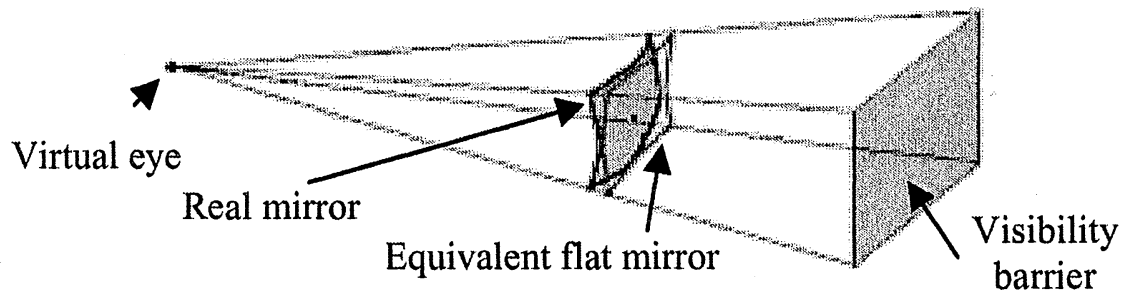

Figure 8. Equivalent flat mirror

In this work, a flat mirror is defined as equivalent to a spherical mirror when its visibility equals that of the spherical mirror; the vertexes of the edges of the equivalent spherical mirror may be derived on the basis of the degree of aberration. The routine converges when the barrier projection is either wholly contained inside the mirror or when it extends beyond the mirror.

Step 3. Modelling the visibility barriers. At this point, the standard visibility barriers can be automatically modelled at various distances from the rear of the vehicle according to the design requirements. They can be enlarged by 5,10 , or $15 \%$ along the vehicle's transversal motion axis to simulate even greater visibility conditions. For example, although the standards prescribe that the barrier for an internal mirror should be located at 
$60 \mathrm{~m}$ from the driver's eyes, the program automatically calculates the dimensions for other distances. To provide the maximum rear visibilitywhich is especially important for trucks and vans manoeuvring in reversefive additional barriers can be created at $30,20,15,12$, and $10 \mathrm{~m}$ from the driver's eyes (Carfagni and Landi 2000). Lastly, since the automatic orientation routines are based only on mirror-barrier positions and are thus unable to predict interposition(s) of obstacles such as the headrest between the mirror and the visibility barrier, the designer has the possibility of manually rotating the mirror. Vertical and horizontal coloured strips of known height are provided on the barrier ends to aid in accounting for the distance between the vehicle and the barrier (Figure 9).

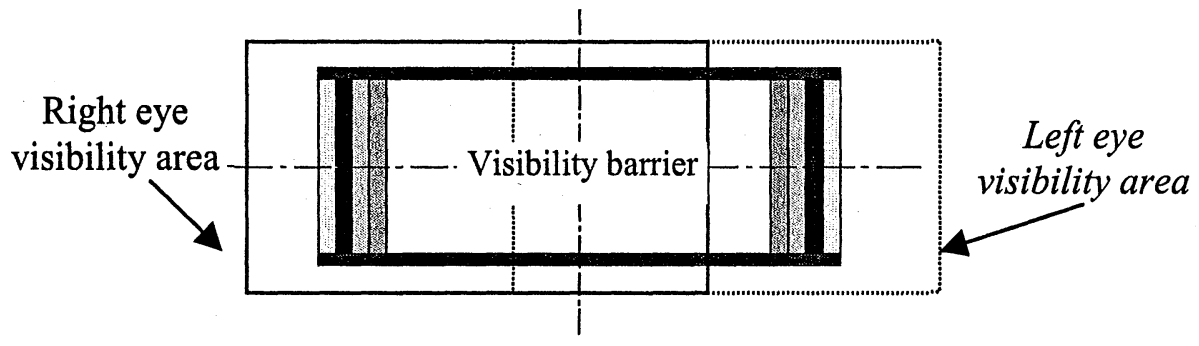

Figure 9. Visibility barrier and visibility areas

By counting the number of strips that are not visible, the designer can determine the amount of displacement (and thus rotation) required to obtain a full view of the barrier.

Step 4. Simulating visibility. The procedure for creating the test environment (including, for example, mirror textures) is described in the User's Manual. The final renderings obtained for tests conducted on the driver's left and right eyes for an internal mirror of a popular automobile are illustrated in Figures 10 and 11.

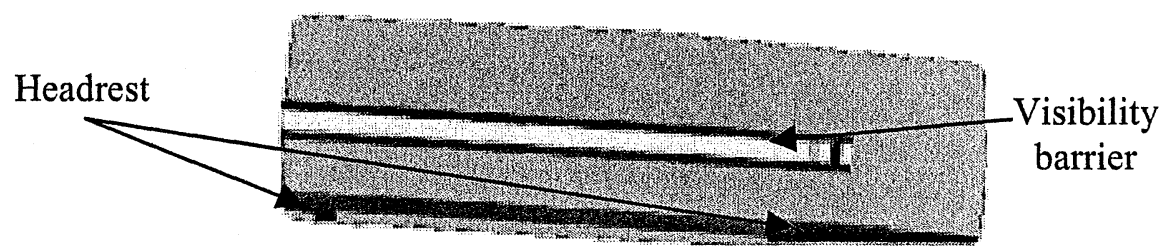

Figure 10. Final left eye rendering for an internal mirror visibility test

The renderings were obtained in a matter of seconds on a Sun Ultra 1 Creator 3D workstation. (Longer calculation times would have been unacceptable because the designer needed six renderings to simulate a complete set of mirrors). 


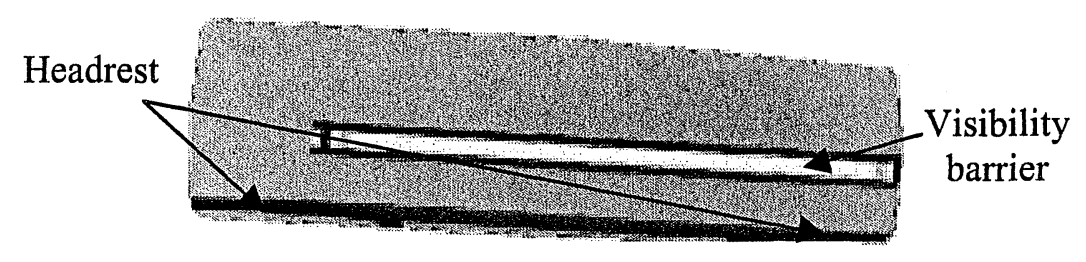

Figure 11. Final right eye rendering for an internal mirror visibility test

Step 5. Performing special tests. The proposed method provides the possibility of conducting additional tests as the special test for van and truck rear visibility, the heated rear windows test and others (Carfagni and Landi 2000).

Truly drivers of vans and trucks often have to make difficult manoeuvres in reverse gear during loading and unloading operations. Although no minimum parameters for rear visibility are indicated in EU standards, this parameter is a prime factor in avoiding accidents such as fender benders, which usually do not cause harm to passengers, but which can cause serious damage to vehicles. So an additional test, that can be activated from the main routine window, enables the designer to quantitatively determine the rear visibility of any vehicle, including vans and trucks. During the first modelling phase, visibility barriers of increasing distance from the vehicle's rear end are added at ground level (at 30,20,15, 12, and 10 meters).

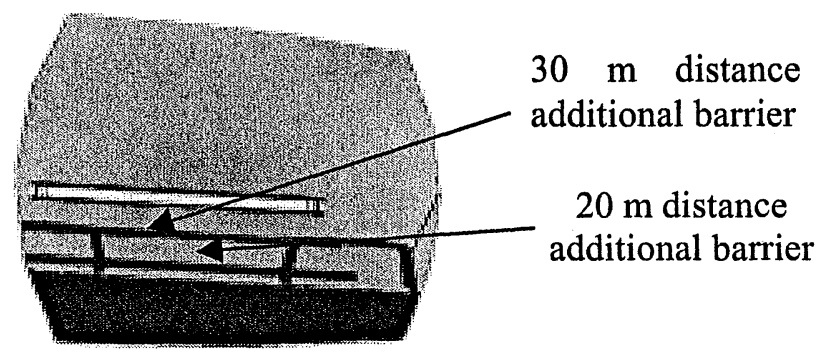

Figure 12.30 and 20 meter barriers added to standard visibility test results

The barriers enable the designer to rapidly determine the test vehicle's minimum rear visibility distance. In Figure 12, a standard visibility test for an inside flat mirror, only the extra 30 and 20 meter barriers are shown, so the "real" ground visibility for the driver is between 20 and 15 meters.

Other CAD-modelled parts may also be tested. For example, international standards mandate that a part of the rear shield area be covered by the heated rear window (defroster). The designer can test rear window visibility by specifying the test from the data input window. This is accomplished by clicking on the surface identifying the rear shield so that 
the application can automatically draw the outer edge of the minimum area required by the standards for the defroster, thereby enabling the designer to select a larger heating area on the rear shield (Figure 13).

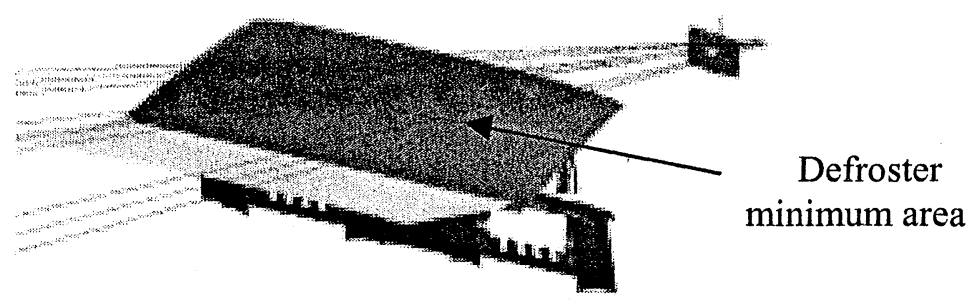

Figure 13. Defroster test

Step 6. Saving and storing data. CAD procedures are expected to output reliable data and standardised reports during the design/simulation phaseyet if the choice of how to save the simulation data were up to the designer, the information might not be easily legible and identifiable even within the manufacturer's own company. Hence, we developed a simple Catia routine, written in $\mathrm{C}$, that performs the following tasks:

- It renders the right and left eye images for the selected test mirror.

- It saves the test vehicle CAD model, the text files with the test input data, and two files with the right- and left eye test mirror images in a user selected directory.

All the operations needed to interact with this program are illustrated in a special section of the User's Manual.

\section{CONCLUSIONS}

The capability of the proposed method to automatically model and test symmetrical rearview mirrors was validated by simulation testing. Its outstanding features include:

- It allows designers to test both flat and spherical mirrors without requiring perspective geometric modelling to simulate the visibility of spherical mirrors in keeping with applicable standards; the innovative use of the ray-tracing modulus and the mirror textures incorporated into the software output correct optical results.

- It allows designers to identify visibility problems caused by obstacles caused by the car body and the interior furnishings in-between the rearview mirror and the test barrier. 
- It allows designers, even without specific training, to quickly develop numerous components which, despite their simplicity, would otherwise require knowledge of the underlying optical laws.

- It allows designers to accurately simulate asymmetrical mirrors using the same procedure with only slight modification.

- It allows designers to rapidly conduct testing not prescribed by current standards simply by rotating the mirror on its joint and repeating the rendering module.

The limits encountered in testing stem from the imperfect integration of the rendering module within the program forcing the designer to execute several commands before proceeding to the actual rendering operations and must therefore be considered inevitable. The method is currently being used by an automobile manufacturer for rearview mirror design and testing.

\section{REFERENCES}

Bom, M. and Wolf, E. , Principles of Optics, 6th Edition, Pergamon Press, New York, 1980.

Carfagni, M. and Landi, L., A Practical CAD-CAE Application for the Automated Design and Testing of Vehicle Rearview Mirrors, Proceedings, 9th International Conference on Machine Design and Production METU, Ankara, Turkey, 2000.

Carfagni, M., Landi, L., Pierini, M., and Poggi, M., Studio di procedure per la simulazione della visibilità posteriore di un autoveicolo, Proceedings, 2nd Congress of the Associazione di Disegno Meccanico, Vico Equense, Salerno, Italy, 1997, "in Italian".

FiatAuto, Disegni CAD: parametri di settaggio per i sistemi CAD, In-house Standards, Turin, Italy, 1997.

FiatAuto, Rearview Mirror Field of View in Passenger Cars, In-house Standards, Turin, Italy, 1986.

IBM, Catia Solution-Softcopy Collection Kit for FrameViewer, Version 4, Release 1.9, Poughkeepsie, NY, USA, 1997.

Kolb, C., Mitchell, D. and Hanrahan, P., A Realistic Camera Model for Computer Graphics, Computer Graphics, Vol. 29 (3), 1995, 317-324

Luneberg, R.K., Mathematical theory of optics, advanced instruction and research in mechanics, Brown University, Providence. RI, 1944.

Nagata, S., The Binocular Fusion of Human Vision on Stereoscopic Displays-Field of View and Environmental Effects, Ergonomics, 1996, Vol. 39.

Sivak, M., Flannagan, M. J., Budnik, E. A., Flannagan, C. C., Kojima, S., Locations of headlamps and driver eye positions in vehicles sold in the USA, Ergonomics, 1007, Volume 40, Issue 9, Pages 872-878

Wheatley, M., Faster time to more money?, Manufacturing Computer Solutions, 1999, Volume 5, Issue.

$\mathrm{Su}, \mathrm{D}$., Design automation with the aids of multiple artificial intelligence techniques, Conc. Engineering Research and Applications, 1999, Volume 7; Issue 1, Pages 23-30 